\title{
Feasibility study for a new high resolution Thomson scattering system for the ASDEX Upgrade pedestal
}

\author{
M. Tsalas ${ }^{a}$, M, Yu. Kantor ${ }^{a, b, c}$, O. Maj ${ }^{d, e}$, R. Bilato ${ }^{d}$, P.C. de Vries ${ }^{a}$, A.J.H Donné ${ }^{a, f}$, \\ A. Herrmann ${ }^{d}$, B. Kurzan ${ }^{d}$, E. Wolfrum ${ }^{d}$ and the ASDEX Upgrade Team ${ }^{d}$ \\ ${ }^{a}$ FOM-Institute for Plasma Physics Rijnhuizen, Association EURATOM-FOM, P.O. Box 1207, 3430 \\ BE Nieuwegein, The Netherlands ${ }^{\mathbf{1}}$ \\ ${ }^{b}$ Institute of Energy and Climate Research - Plasma Physics, Forschungszentrum Jülich GmbH, \\ Association EURATOM-FZJ, D-52425 Jülich, Germany \\ ${ }^{c}$ Ioffe Institute, RAS, Saint Petersburg 194021, Russia \\ ${ }^{d}$ Max-Planck-Institut für Plasmaphysik, Association IPP-EURATOM, D-85748 Garching, Germany \\ e Max-Planck-Institut für Sonnensystemforschung, D-37191, Katlenburg-Lindau, Germany \\ ${ }^{f}$ Applied Physics Department, Eindhoven University of Technology, The Netherlands \\ E-mail:m.tsalas@rijnhuizen.nl \\ - Partners in the Trilateral Euregio Cluster
}

\begin{abstract}
A new Thomson scattering diagnostic is proposed for the study of fast plasma dynamics in the pedestal of ASDEX Upgrade. The diagnostic will measure electron temperature and density profiles over a $\sim 3 \mathrm{~cm}$ wide area in the edge transport barrier region, with $\sim 1-2 \mathrm{~mm}$ spatial resolution and $\sim 10 \mathrm{kHz}$ sampling rate. A challenging goal of the project is the study of the bootstrap current in the plasma pedestal by measuring the distortion and shift of the electron distribution along the toroidal direction. Expected spatial and time resolutions of the current density measurements are $\sim 3 \mathrm{~mm}$ and $\sim 1 \mathrm{~ms}$ correspondingly. The new diagnostic will be used to study the fast dynamic behaviour of the pedestal bootstrap current, where models indicate that it plays a key role in regulating edge stability, e.g. during ELMs. The diagnostic design is based on the intra-cavity multi-pass system currently in operation in TEXTOR, which uses a probing ruby laser, a grating spectrometer and two fast CMOS cameras for scattered light detection, and has achieved measuring accuracies of the order of $\sim 1 \%$ for $n_{e}$ and $\sim 2 \%$ for $T_{e}$. Parts of that system will be reused in ASDEX Upgrade (some with significant modifications), but the laser multi-pass and light collection systems are entirely redesigned. Restrictions in space and line-of-sight availability have led to the adoption of a design which uses in-vessel multi-pass mirrors and light collection optics, requiring a number of innovative technical solutions to permit remote laser alignment and light collection. We give an overview of the project, discuss the underlying physics basis and present a number of technical solutions employed.
\end{abstract}

KEYWORDS: Nuclear instruments and methods for hot plasma diagnostics; Plasma diagnostics interferometry, spectroscopy and imaging 


\section{Contents}

\section{Introduction}

\section{Conditions in the ASDEX Upgrade pedestal}

3. Section Required measurement accuracy

\section{The TEXTOR multi-pass TS system}

5. The proposed ASDEX Upgrade scheme

6. Measurement accuracy estimate

\section{Conclusions}

\section{Introduction}

The plasma current density $j$ has been recognised as an important parameter in tokamak reactor performance optimization. In the plasma core, the $j$ profile affects both the confinement and the MHD stability. At the plasma edge, existing models [1] indicate that it plays a crucial role in the regulation of the periodic edge profile relaxations known as Edge Localised Modes (ELMs) that appear when a spontaneous edge transport barrier forms above a heating power threshold, resulting in improved confinement (what is known as the H-mode regime). In a tokamak-based fusion device, the stability limit of this edge transport barrier will define the performance. ELMs could also cause damaging transient heat loads onto the divertor target plates. Understanding and mitigating ELMs is therefore one of the key challenges in fusion research.

The framework which seems to best explain the ELM onset is a linear MHD model referred to as the peeling-ballooning model [1], which describes the interaction between two classes of edge-localized, ideal MHD instabilities: peeling modes, low toroidal number $n$ external kink instabilities driven by edge current and stabilized by pressure gradient and magnetic shear, and high $n$ ballooning modes driven by the pressure gradient. This model sets limits on the edge pressure gradient $\nabla p$ and parallel current density $j_{\|}$. Exceeding these limits is believed to trigger an ELM, thereby relaxing the instability drive. It is clear that in such a model, the current density at the edge plays a fundamental role. Nevertheless, $j$ is often only calculated from the pressure gradient using neoclassical theory. A robust, systematic and nonintrusive way of measuring the edge $j$ in present tokamaks remains elusive, hampering full experimental validation of the peeling-ballooning model.

In general, edge current density measurements in tokamaks are not straightforward. A number of diagnostic methods have been employed until now to measure edge $j$, e.g. from the Zeeman splitting of intrinsic or extrinsic impurities [2], from Motional Stark Effect (MSE) polarimetry [3] and from electron Bernstein wave emission [4]. Such methods are indirect in that they rely on measurements of the poloidal field $B_{\theta}$, from which the generating current can then be calculated. Each of these methods has advantages and disadvantages, ranging from the fact that they only provide line-integrated measurements that require inversion, to significant 
technical complications in their implementation. The successful use of MSE polarimetry in MAST is for example aided by the large magnetic field pitch angle and small radial electric field at the edge, conditions which are particular to spherical tokamaks.

Thomson scattering (TS) with a tangential viewing line on the other hand allows direct measurement of the electron velocity distribution function, $f(v)$, from which the current density:

$$
j=e\left(n_{i} v_{d, i}-n_{e} v_{d, e}\right),
$$

can then be calculated, assuming the ion density, $n_{i}$, and the ion drift velocity, $v_{d, i}$, are also measured in parallel, e.g. by charge exchange recombination spectroscopy [5]. $n_{e}$ and $v_{d, e}$ are the electron density and electron drift velocity respectively, and the electronic part of the current density is then given by [6]:

$$
j_{e}=e n_{e} v_{d, e}=e \int_{-\infty}^{+\infty} f(v) v d v .
$$

Measurements of $j_{e}$ have already been demonstrated at the core (e.g. [7], [8], [9]), but with a spatial and temporal resolution nowhere near the requirements needed to study fast ELM events. The main obstacle is that extremely high accuracy is required in order to make possible the separation of the electron drift velocity component $v_{d, e}$ from the thermal velocity $v_{t h, e}$. Typically, for a streaming parameter $\xi=v_{d, e} / v_{t h, e} \sim 0.05$, determination of $v_{d, e}$ within a $\sim 20 \%$ accuracy requires an accuracy of less than $1 \%$ on $v_{t h, e}$ (more on this in section 3 ). This level of precision requires not only very high photoelectron statistics (i.e. high laser energy, high detector efficiency), but also elimination of other sources of error, such as plasma light, stray light, and very accurate calibration.

In this paper we will report on a feasibility study performed for a new tangential Thomson scattering diagnostic for ASDEX Upgrade. In addition to standard $n_{e}$ and $T_{e}$ profiles, the new system will also aim to provide fast profile measurements of $j_{e}$. The diagnostic will be based on the intra-cavity multi-pass system developed at TEXTOR [10], which has demonstrated measurement capabilities well within the desired range for current density determination. Section 2 gives a brief overview of the plasma conditions in the ASDEX Upgrade pedestal. Section 3 provides a crude estimate of the accuracy such a system must achieve. Section 4 describes the TS system currently in operation at TEXTOR, on which the proposed ASDEX system will be based. The proposed diagnostic setup is discussed in section 5. Section 6 presents calculations for the expected edge current measurement accuracy.

\section{Conditions in the ASDEX Upgrade pedestal}

A characteristic ASDEX Upgrade H-mode edge density and temperature profile just before an ELM crash is shown in figure 1 (pulse \#23227, $I_{p}=1 \mathrm{MA}, B_{t}=2.5 \mathrm{~T}$, line - integrated density $<n_{e}>\sim 6.5 \cdot 10^{19} \mathrm{~m}^{-3}$ ).

Typical electron densities range from $n_{e} \sim 0.5 \cdot 10^{19} \mathrm{~m}^{-3}$ at the bottom of the pedestal to $n_{e} \sim$ $5 \cdot 10^{19} \mathrm{~m}^{-3}$ at the top. Similarly, the electron temperature range is $T_{e} \sim 0.1-1 \mathrm{keV}$. The pedestal width is of the order of $1.5-2.5 \mathrm{~cm}$ and the ELM frequency $\sim 80-150 \mathrm{~Hz}$, thus requiring a minimum diagnostic spatial resolution of $\sim 3-5 \mathrm{~mm}$ and a temporal resolution of at least $1-2$ ms for reasonable ELM characterisation. Modelling of the edge bootstrap current (which is the main component of the total edge current) with the CLISTE code [11] has given a broad range of values for $j$ at the mid-plane, ranging from $\sim 0.5$ to $4 \mathrm{MA} / \mathrm{m}^{2}$ (see e.g. figure 3 in [12]). The plasma rotation in the vicinity of the separatrix is of the order of $15-30 \mathrm{~km} / \mathrm{s}$ [5], which gives a range for $v_{d, i} / v_{d, e} \sim 0.02-0.4$, so that in most cases $v_{d, i}$ cannot be neglected in the calculation of 
$j$. The streaming parameter range is $\xi=v_{d, e} / v_{t h, e} \sim 0.005-0.06$. The poloidal magnetic field is of the order of $0.35-0.4 \mathrm{~T}$.

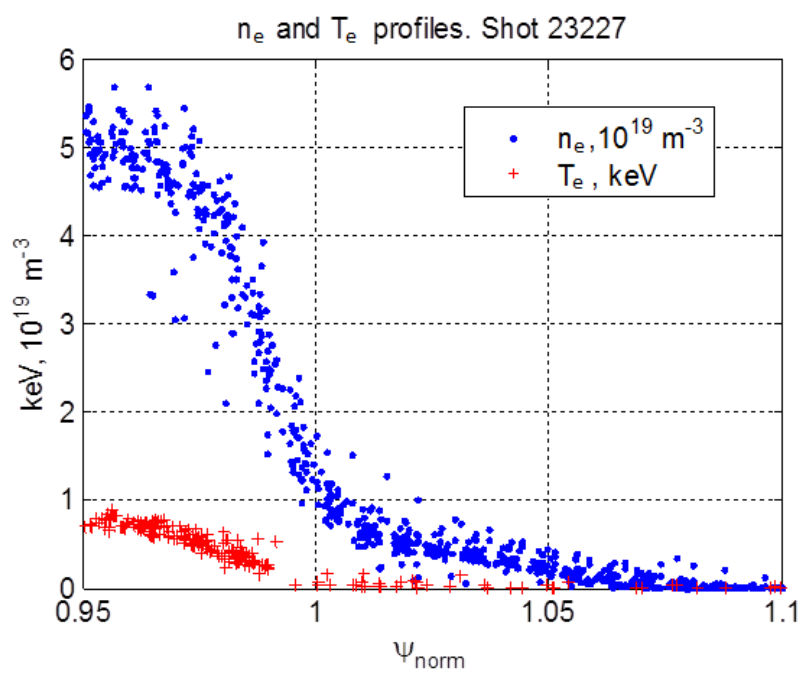

Figure 1: Electron temperature and density profiles in the ASDEX Upgrade pedestal, measured with ECE and Li-beam respectively.

\section{Required measurement accuracy}

A rough first estimate of the required accuracy can be obtained as follows: when measuring tangentially to the magnetic field, $j_{e}$ will correspond to an electron drift velocity $v_{d, e}$, which will cause a Doppler shift in the measured scattered spectrum. This spectral shift is given by [9]:

$$
\Delta \lambda_{d}=\frac{2}{c} \lambda_{0} v_{d, e} \sin \frac{\theta}{2} \sin \left(\phi-\frac{\theta}{2}\right) \cos \gamma,
$$

where $\lambda_{0}$ is the wavelength of the incoming beam $(=694.3 \mathrm{~nm}$ for a Ruby laser), $\theta$ is the scattering angle (taken here to be $90^{\circ}$ ), $\gamma$ is the angle between the scattering plane and the drift velocity (in the proposed tangential geometry, $\cos \gamma \sim 1$ ), $\phi$ is the angle between the incoming beam and the projection of the drift velocity vector onto the scattering plane (again $\phi \sim 90^{\circ}$ in the proposed geometry) and $c$ is the speed of light in vacuum. The electron drift velocity can be estimated by $v_{d, e}=j_{e} / e n_{e}$ which, for the values of $j_{e}$ and $n_{e}$ given in the previous section, yields $\Delta \lambda_{d} \sim 1.6 \mathrm{~nm}$. This should be compared with the thermal width of the scattered spectrum, given by $\Delta \lambda_{e}=\left(2 \lambda_{0} v_{t h, e} \sin \theta / 2\right) / c \sim 20-65 \mathrm{~nm}$, where $v_{t h, e}=\sqrt{2 e T_{e}(e V) / m_{e}} \sim 6 \cdot 10^{6}-$ $2 \cdot 10^{7} \mathrm{~m} / \mathrm{s}$. Therefore, the determination of $\Delta \lambda_{d}$ with an error of $\pm 20 \%$ (corresponding to \pm 0.3 $\mathrm{nm}$ ) would require a measurement accuracy for $\Delta \lambda_{e}$ of the order of $0.4-1.5 \%$ (for $\Delta \lambda_{e}=65$ and $20 \mathrm{~nm}$ respectively).

\section{The TEXTOR multi-pass TS system}

The very high experimental accuracy needed for the determination of $j$ requires a TS system with extremely high diagnostic performance, i.e. high measured photoelectron statistics and also very low instrumental errors. Methods for the minimization of additional errors caused by plasma light, stray light and calibration or detector issues must also be carefully 
implemented. For the investigation of ELMs, all this must also be combined with good spatial and temporal resolution. In this respect, the TEXTOR multi-pass TS system (for details, see [10]), shown in figure 2, has demonstrated that such a level of accuracy is within reach, e.g. by measuring spectral shifts of $\sim 0.5 \mathrm{~nm}$, even though measured at an angle of $\sim 81^{\circ}$ with respect to the toroidal direction [13]. In that system, intra-cavity probing of the plasma with a repetitively pulsed Ruby laser is combined with a multi-pass system which multiplies the probing energy, and a fast (10 kHz), 12-bit, 512x384 pixel CMOS detection camera measuring the scattered light and the background light (for subtraction) in alternation. The system routinely achieves $5 \mathrm{kHz}$ repetition rate for $8 \mathrm{~ms}$, with a probing energy of $\sim 50 \mathrm{~J}$ per pulse, providing $n_{e}$ and $T_{e}$ profiles with a $\sim 1 \mathrm{~cm}$ spatial resolution across a $\sim 0.9 \mathrm{~m}$ long chord and measurement accuracy of the order of $\sim 1 \%$ for $n_{e}$ and $\sim 2 \%$ for $T_{e}[14]$.

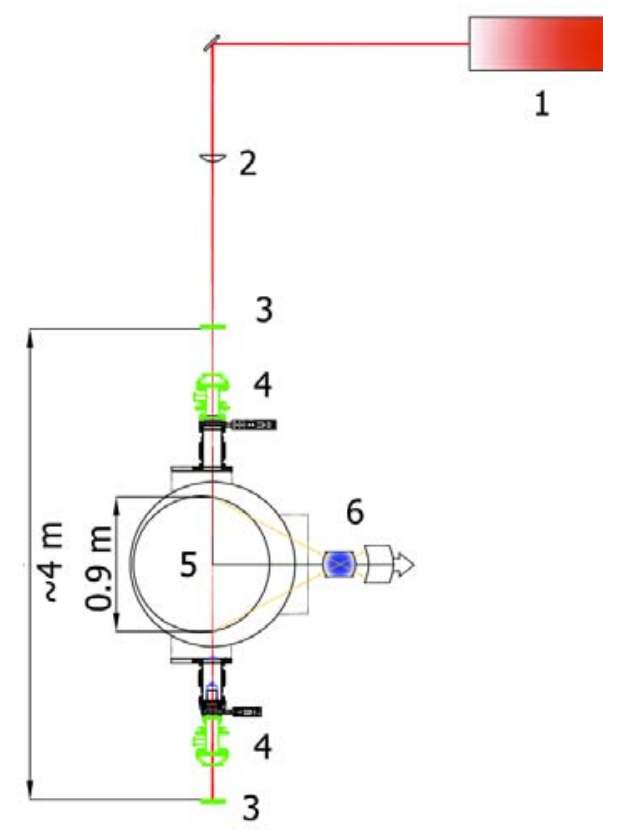

Figure 2: The experimental setup of the TEXTOR multi-pass TS system: 1) Ruby laser, 2) focusing lens, 3) spherical mirror, 4) Brewster window, 5) plasma and 6) collection objective and fiber array input.

\section{The proposed ASDEX Upgrade scheme}

A number of the technical solutions employed in TEXTOR can also be used for the proposed ASDEX Upgrade system. In fact, some of the TEXTOR components will be re-used in ASDEX Upgrade, after undergoing maintenance and upgrades. The principle components which will be re-used are the Ruby laser system, with its power supply and control units, and the data collection unit consisting of the spectrometer stage and light detection system, including the grating spectrometer, image intensifier and one CMOS camera. The laser power supply system will be upgraded to allow four (instead of one) laser bursts, and therefore extending the measurement time in comparison with the existing system. In addition, a second CMOS camera will be installed, doubling the frame acquisition rate to $20 \mathrm{kHz}$. In other respects however, these parts of the diagnostic will remain identical to the existing TEXTOR system (to begin with at least - further upgrades might be considered at a later stage). 
For the ASDEX Upgrade system however, restrictions in space availability and also the required radial resolution do not allow application of a TEXTOR-like multi-pass configuration, and therefore another type of multi-pass system will be used [15]. This is shown in figure 3. This setup consists of 32 passes between two in-vessel glass spherical mirrors with dielectric coating, that create a laser "fan" with a total cavity length of $\sim 50 \mathrm{~m}$. The radial thickness of the fan at the mid-plane is $\sim 3 \mathrm{~cm}$, spanning the ASDEX Upgrade pedestal region. The top mirror will have a hole in the centre, from which the laser beam will enter and exit. The short distance between the two mirrors $(\sim 1.4 \mathrm{~m})$ and the required mirror curvature will assure good optical stability. In addition, the mirrors will be fixed onto two internal passive coil structures (shown in yellow in figure 3), which are rigidly connected to each other and do not vibrate strongly. This setup will ensure that the susceptibility of the laser cavity alignment to vessel vibrations is minimised.

The mirrors have to be aligned between tokamak shots with the use of remotely controlled piezo motors, themselves protected from plasma exposure by a shield. The coated reflecting surfaces of the mirrors have also to be protected from plasma pollution by shutter plates, which will open only during the laser burst. An alignment procedure similar to the one used in TEXTOR will be utilised (adapted of course to the different setup) and executed entirely remotely. For this, an in-vessel video monitoring system will be installed, to look at spots created by a pilot laser on the mirrors, which will guide the diagnostic operator in aligning the system.
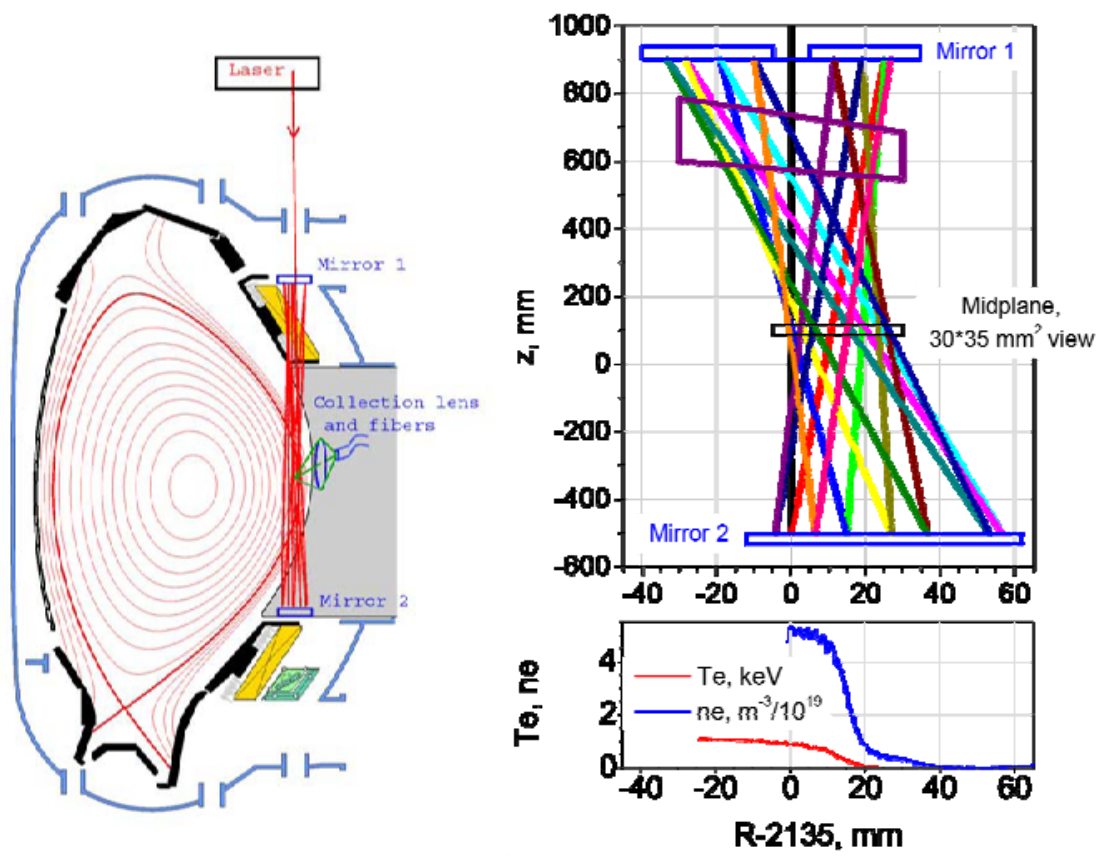

Figure 3: Left: Poloidal cross-section of ASDEX Upgrade, with the proposed setup for the new TS system. Right: The multi-pass configuration and relative pedestal position. The beam enters from a hole on the top mirror, from which it exits again after 32 passes through the plasma (16 passes shown, after which the beam retraces the same path backwards).

The collection optics will also be positioned fully in-vessel, and will consist of an F/2.5, 40 $\mathrm{mm}$ diameter collection objective and a $\sim 4.5 \mathrm{~m}$ long fibre bundle, which will carry the optical signal across a window to the spectrometer unit. The fibre bundle will consist of $\sim 2000$ pure fused silica fibres of $\sim 300 \mu \mathrm{m}$ diameter, protected by E-glass and stainless steel sleeves. The 
bundle ends will be glued on stainless steel ferrules by epoxy. The entire bundle will be high vacuum compatible (up to $10^{-11} \mathrm{mbar}$ ) and able to withstand baking temperatures of up to 200 ${ }^{\circ} \mathrm{C}$. The scattered light will be collected at an angle of $\sim 18^{\circ}$ with respect to the toroidal tangent, from a region $\sim 30 \mathrm{~mm}$ wide on the tokamak mid-plane, overlapping with the pedestal region (figure $3 \mathrm{~b}$ ). Profile characterisation is realised by scattered light measurement across the beam fan, rather than the more usual setup of light collection along the laser beam.

Beam-tracing calculations (assuming a Gaussian shaped laser beam and realistic beam divergence) of the expected probing energy density along the mid-plane are shown in figure 4. It is seen that with the proposed configuration an average linear energy density of $\sim 7 \mathrm{~J} / \mathrm{mm}$ can be achieved across the probing region, the minimum being $\sim 3 \mathrm{~J} / \mathrm{mm}$ in the centre of the fan. The height of the observation volume will depend on the etendue of the collection optics. For a collection solid angle of $\Omega \sim 0.01 \mathrm{sr}$ and assuming an etendue similar to the TEXTOR system ( $6.8 \mathrm{~mm}^{2} \cdot \mathrm{sr}$ ), a height of $\sim 22 \mathrm{~mm}$ is foreseen.

A maximum radial resolution of $\sim 1.5 \mathrm{~mm}$ is envisaged, limited by the pixel cross-talk of the CMOS camera. However, by averaging signals over neighbouring spectral channels, improved measurement statistics can be obtained, since a $3 \mathrm{~mm}$ spatial resolution is still sufficient for profile characterisation. In the same way, by averaging data over 5-10 laser pulses, the measurement statistics can be improved even more. Since the laser can be pulsed at a 10 $\mathrm{kHz}$ rate, this would give a temporal resolution of $0.5-1 \mathrm{~ms}$, again sufficient for fast ELM characterisation.

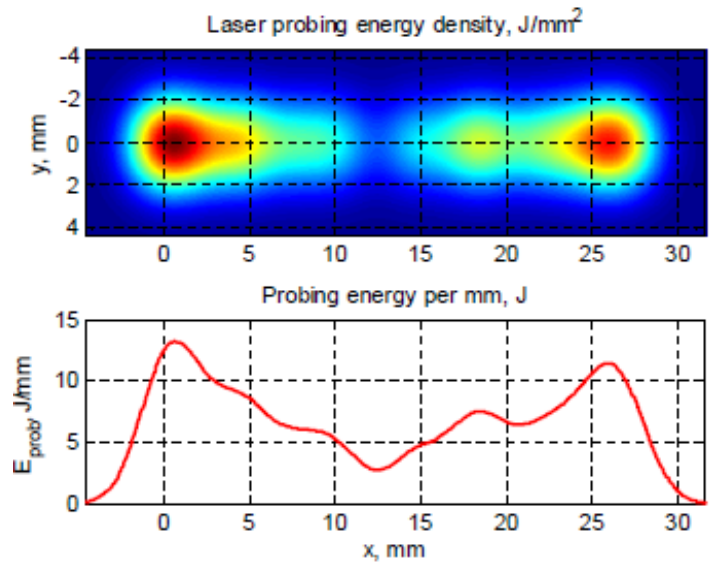

Figure 4: Calculated laser probing energy at the mid-plane across the laser fan.

\section{Measurement accuracy estimate}

Calculations were performed in order to determine the expected measurement accuracy in the proposed configuration [16]. As a first step, a simplified model was used, which neglects relativistic and high temperature effects. This assumption is not necessarily fully justified in this case, but it is a first step on which relativistic effects can be subsequently added.

Starting from the total number of photoelectrons measured by the detector in a spectral channel of frequency width $d \omega_{s}$ centred around the frequency $\omega_{s}$, which is given in case relativistic effects are neglected by:

$$
N_{s c}\left(\omega_{s}\right)=\eta n_{e} \frac{E_{i}}{\hbar \omega_{i}} r_{0}^{2} L \Delta \Omega k^{-1} d \omega_{s} h\left(\frac{\Delta \omega}{k}\right),
$$


where $\omega_{s}$ and $\omega_{i}$ are the scattered and incident beam angular frequencies respectively and $\Delta \omega=$ $\omega_{s}-\omega_{i}, \eta$ is the overall measurement efficiency, including the quantum efficiency of the detector and the efficiencies of the transmission and collection optical lines, $E_{i}$ is the total laser energy during a pulse, $r_{0}$ is the classical electron radius, $L$ is the height of the scattering region, $\Delta \Omega$ is the full solid angle of the collection optics, $d \omega_{s}$ is the width of each detector spectral channel, $k$ is the magnitude of the differential wave vector $\boldsymbol{k}=\boldsymbol{k}_{\boldsymbol{s}}-\boldsymbol{k}_{\boldsymbol{i}}$, with $k=2 \omega_{i} \sin (\theta / 2) / c$ and the function $h$ is the projection of the electron velocity distribution function $f(\mathbf{v})$ along the direction of $\boldsymbol{k}$, given by:

$$
h\left(v_{k}\right)=\frac{1}{n_{e}} \int_{-\infty-\infty}^{\infty} \int_{-\infty}^{\infty} f\left(v_{x}, v_{y}, v_{k}\right) d v_{x} d v_{y},
$$

with $v_{k}=\Delta \omega / k$ and the $\mathrm{z}$ axis was chosen such as $\hat{z}=\hat{k}$.

The z-component of the electron current density is then given by the first-order moment of the velocity distribution function, which can be written from equations (2), (4) and (5) as:

$$
j_{e}=\frac{e \hbar \omega_{i}}{\eta E_{i} r_{0}^{2} L \Delta \Omega} \int \frac{\Delta \omega}{k} N_{s c, \omega}\left(\omega_{s}\right) d \omega_{s},
$$

where $N_{s c, \omega}$ is the number of measured photons per unit frequency (we used $N_{s c}=N_{s c, \omega} d \omega_{s}$ ). By approximating the integral as a sum over all spectral channels, one obtains:

$$
j_{e}=\frac{e c \hbar \omega_{i}}{\sqrt{2} \eta \sin (\theta / 2) E_{i} r_{0}^{2} L \Delta \Omega} \sum_{\text {channels }} \frac{\Delta \omega}{\omega_{i}} N_{s c}\left(\omega_{s}\right),
$$

where it is possible to show that, for the spectral channel width to be considered here, the error associated with the discretisation is $\sim 21.4 \%$ for a spectral band corresponding to 1.5 thermal widths. This can be however significantly improved by increasing appropriately the measurement bandwidth, and can be made small compared to the main sources of measurement error, i.e. errors due to Poisson fluctuations and also due to the background plasma light, mainly Bremsstrahlung. The total error in $j_{e}$ associated with these can be obtained by taking the standard deviation of the statistical distribution of the contribution of each channel to the current density. The standard deviation of the current density is then obtained as the square root of the sum of variances of each channel, assuming that channels are statistically uncorrelated. This yields:

$$
\Delta j_{e} / j_{e}=\frac{\sqrt{\sum \Delta \omega^{2}\left(N_{s c}+2 N_{b}\right)}}{\sum \Delta \omega N_{s c}},
$$

where $N_{b}$ is the number of Bremsstrahlung photoelectrons collected by the detector (see equations (3) and (4) in [17]), and the two sums are over all spectral channels as before. This relationship can be thought of as the result of an ideal measure, placing a lower bound on the actual measurement error.

As a next step, a shifted Maxwellian velocity distribution function was used to calculate $\Delta j_{e}$ $/ j_{e}$ as a function of the streaming parameter $\xi$. The shifted Maxwellian was chosen because it arguably provides a better approximation to the effect of the bootstrap current on the electron velocity distribution function. For this calculation, the plasma parameters used correspond to the middle of the pedestal region shown in figure 1, i.e. $n_{e} \sim 2.5 \cdot 10^{19} \mathrm{~m}^{-3}, T_{e} \sim 0.5 \mathrm{keV}$, the total diagnostic efficiency was taken to be the same as for the TEXTOR system, i.e. $\eta \sim 0.03$, the 
laser pulse energy $E_{i}=20 \mathrm{~J}$ (for a spatial resolution of $\sim 3 \mathrm{~mm}$ ), $d \omega_{s} \approx \frac{2 \pi c}{\lambda_{i}^{2}} d \lambda_{i}$ with $\lambda_{i}=694.3$ $\mathrm{nm}$ for a Ruby laser and $d \lambda_{i}=2 \mathrm{~nm}$ (the spectral channel width), a spectral bandwidth corresponding to 1.5 thermal widths and geometrical parameters similar to the geometry presented in section 5. The total number of registered photoelectrons in the calculation was 88000 . We also used a more realistic value for the background plasma light, namely $N_{b} \sim N_{s c / a v} /$ 10 ( $N_{\text {sc/av }}$ being the mean scattered light intensity over the full thermal band), taking as a basis what is typically measured at TEXTOR for similar plasma parameters (see e.g. figure 8 in reference [10]). In addition, the errors can be improved by elongation of the gate for plasma light measurements. With a longer measurement gate, more accurate measurements of plasma light can be achieved and, as a result, the term $2 N_{b}$ in (8) would tend to $N_{b}$ (we used this in the calculation).

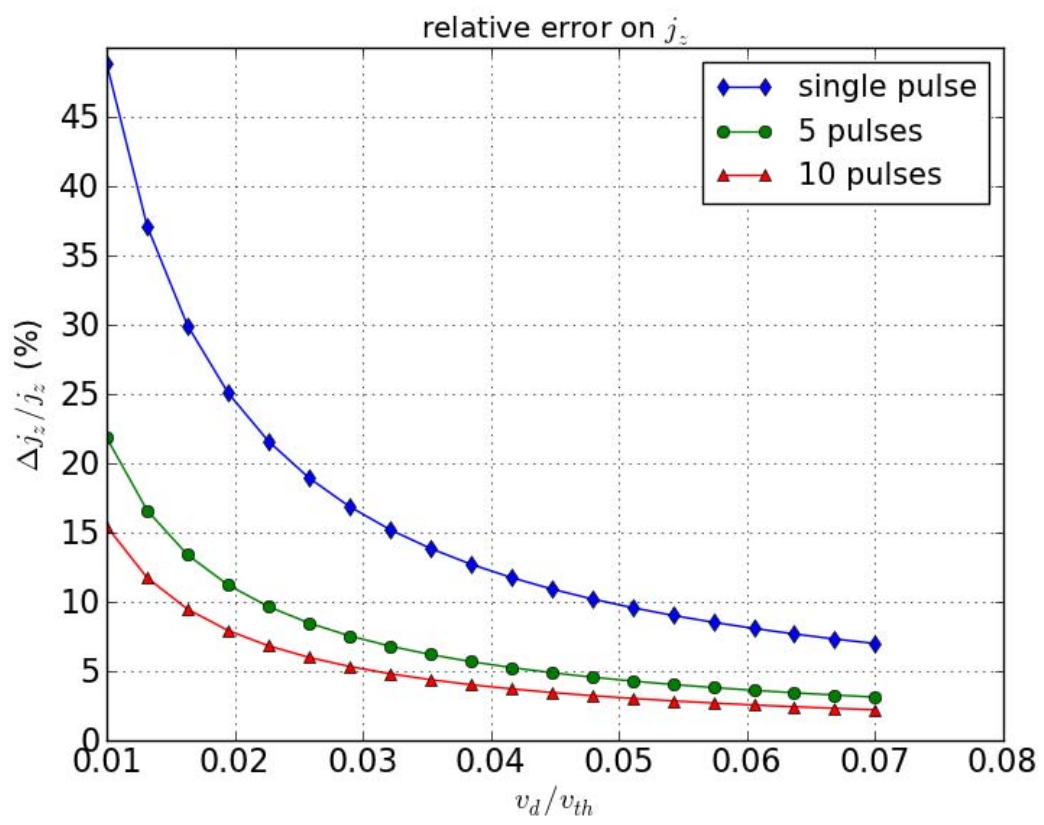

Figure 5: Relative error on the simulated measure of the plasma electric current density according to equation (8), using a shifted Maxwellian velocity distribution function, calculated for 1 (blue), 5 (green) and 10 (red) laser pulses.

The resulting $\Delta j_{e} / j_{e}$ as a function of $\xi$ for a single laser pulse is shown as the blue curve in figure 5. It is observed that the relative simulated error varies significantly with the streaming parameter $\xi$, ranging from above $45 \%$ for $\xi \sim 0.01$ to below $15 \%$ in the region $0.035<\xi<0.07$. This accuracy can be significantly improved by summing over a number of laser pulses. Since the maximum laser pulse repetition rate is $10 \mathrm{kHz}$, it is possible to sum over 5 or even 10 pulses, and still maintain a high enough temporal resolution to study ELMs. The calculated $\Delta j_{e} / j_{e}$ for these two cases is shown in figure 5. The 5 pulse case (green curve) corresponds to a temporal resolution of $2 \mathrm{kHz}$ and the 10 pulse case (red curve) to a temporal resolution of $1 \mathrm{kHz}$. It is seen that even for low values of $\xi(\sim 0.01)$ an accuracy of $15 \%$ can be achieved in this way, while at high enough $\xi$ values, the accuracy can be of the order of 5\%. Although only a lower bound on the actual error, such estimates indicate that a reasonable accuracy in the measurement of $j_{e}$ can be expected, at least for values of $\xi>0.01$, and are therefore encouraging. 
As a final remark in this section it should be noted that a) the above calculation does not include relativistic corrections, which should in principle be applied for $T_{e}>200 \mathrm{eV}$ and will tend to blue-shift the spectrum significantly, and b) the shifted Maxwellian distribution function is only a first approximation of $f$. Ideally, the above analysis should be repeated with the application of a numerical solution to the Fokker-Planck equation instead.

\section{Conclusions}

A feasibility study for the implementation of an intra-cavity multi-pass system in ASDEX Upgrade has been presented. The new diagnostic aims to provide fast current density profile measurements (in addition to the usual $n_{e}$ and $T_{e}$ profiles), and will be used for stability studies in the ASDEX Upgrade edge transport barrier region. The feasibility study has demonstrated that such measurements can be achieved with an intra-cavity multi-pass system implementing many features from the system currently in use in TEXTOR. Expected spatial and temporal resolutions are of the order of $\sim 1.5-3 \mathrm{~mm}$ and $\sim 0.1-1 \mathrm{~ms}$ respectively. Calculations indicate that a measurement accuracy of $\sim 5-15 \%$ for $j$ can be achieved.

\section{Acknowledgments}

This work was supported by NWO-RFBR Centre-of-Excellence grant 047.018.002. This work, supported by the European Communities under the Contract of Association between EURATOM-FOM, was carried out within the framework of the European Fusion Programme. The views and opinions expressed herein do not necessarily reflect those of the European Commission.

\section{References}

[1] J.W. Connor et al., Magnetohydrodynamic stability of tokamak edge plasmas, Phys. of Plasmas 5 (1998) 2687.

[2] K. Kamiya et al., Zeeman polarimetry measurement for edge current density determination using Li-beam probe on JT-60U, Rev. Sci. Instrum., 81 (2010) 033502.

[3] M.F.M. De Bock, Measurements of the edge current during MAST H-modes using MSE, Proc. $37^{\text {th }}$ EPS Conference, Dublin (2010) O2.107.

[4] R.G.L. Vann, Using Bernstein wave emission to measure the current in the tokamak edge, Proc. $37^{\text {th }}$ EPS Conference, Dublin (2010) P5.138.

[5] T. Putterich et al., Evidence for strong inversed shear of toroidal rotation at the edge-transport barrier in the ASDEX Upgrade, Phys. Rev. Lett. 102 (2001) 025001.

[6] I.H. Hutchinson, Current density measurement in Tokamak-type devices by longitudinal Thompson scattering: a proposal, J. Appl. Phys. 10 (1977) L11.

[7] F. Alladio and M. Martone, Thomson scattering measurements from a current carrying plasma and the determination of local current density in a Tokamak (T.T.F.2), Phys. Lett. 60A (1977) 39.

[8] C.J. Barth, Determination of the local current density by means of tangential Thomson scattering: Experimental setup, feasibility test, and preliminary observations, Rev. Sci. Instrum. 60 (1989) 2673. 
[9] F.A. Karelse et al., Measurements of the current density profile with tangential Thomson scattering in RTP, Plasma Phys. Control. Fusion 43 (2001) 443.

[10] M.Yu. Kantor et al., Thomson scattering system on the TEXTOR tokamak using a multi-pass laser beam configuration, Plasma Phys. Control. Fusion 51 (2009) 055002.

[11] P.J. McCarthy et al., The CLISTE interpretative equilibrium code, IPP Report 5/85 (1999), MaxPlanck-Institut fur Plasmaphysik, Garching, Germany.

[12] M.G. Dunne et al., Inter-ELM pedestal current density evolution in ASDEX Upgrade, Proc. $38^{\text {th }}$ EPS Conference, Strasbourg (2011), P-1.107.

[13] M. Kantor, Deviations from Maxwellian electron distribution in the reconnection region of magnetic islands in the TEXTOR tokamak, Proc. $38^{\text {th }}$ EPS Conference, Strasbourg (2011), P-2.083.

[14] M.Yu.Kantor et al., Thomson scattering diagnostic for study fast events in the TEXTOR plasma Proc. 36th EPS Conference, Sofia (2009), ECA Vol.33D, P-1.184.

[15] M.Yu.Kantor et al., Local diagnostics of hydrogen atom and molecular density based on laser induced ionization in FT-2 tokamak, Proc. $13^{\text {th }}$ Int. Symp. on Laser-Aided Plasma Diagnostics. Takayama, Japan (2007) NIFS-PROC-68, p. 104.

[16] O. Maj and R. Bilato, Spectrum of incoherent Thomson scattering diagnostics in current-carrying plasmas, IPP Internal Report, Max-Planck-Institut fur Plasmaphysik, Garching, Germany.

[17] J. Lasalle and P. Platz, Signal-to-noise scaling of ruby laser scattering from large-tokamak plasmas, Plasma Phys. 20 (1978) 107. 